\title{
Abstracts of Papers Presented at the 123rd Meeting of the American Astronomical Society, held 27-30 December 1966 at the University of California at Los Angeles, California
}

A Statistical Study of the Distribution of Clusters of Galaxies. G. O. Abell and Courtney E. SeligMAN, University of California, Los Angeles.-An analysis of the distribution of clusters of galaxies listed in the Catalogue of Galaxies and Clusters of Galaxies, being compiled by Zwicky and his associates, has been extended to include all three volumes (I, II, and V) now published. All clusters at galactic latitudes greater than $30^{\circ}$ are considered. For clusters of each distance category we determine the numbers of clusters in square grid cells with areas ranging from 1 to 196 square degrees. Three statistical tests are performed:

(1) The frequency distribution, $f(t)$ of cells containing $t$ clusters each is compared to the binomial distribution (expected for randomly distributed noninteracting objects) with a $\chi$-squared test.

(2) The ratio of the dispersion about the mean number of clusters per cell to that for the binomial distribution is determined as a function of cell size, and the significance of the difference between the two dispersions is tested with the $F$ distribution.

(3) Serial correlations of counts of clusters in 4 sq deg cells are determined as a function of the cell separation.

All tests reveal extremely significant departures of the observed from the binomial distribution, contrary to results of a recent analysis reported by Zwicky (Z. Astron. 64, 246, 1966). The distribution is consistent with one containing second-order clusters, and is, moreover, not inconsistent with the hypothesis of complete second-order clustering.

Intensity and Linear Polarization Observations of Cassiopeia A, Cygnus A, and Taurus A at $1.97 \mathrm{~cm}$. R. J. Allen AND A. H. Barrett, Massachusetts Institute of Technology.-The 37-m Haystack antenna of the Lincoln Laboratory has been used to map the distribution of antenna temperature and linear polarization of the discrete sources Cassiopeia $\mathrm{A}$, Cygnus A, and Taurus A at $1.97 \mathrm{~cm}(15.25$ $\mathrm{GHz}$, bandwidth $0.5 \mathrm{GHz}$ ). At this wavelength, the antenna beamwidth of $2.18 \mathrm{~min}$ of arc permits partial resolution of the sources. The data have been digitally filtered in a computer to remove those components of spatial frequency which the antenna beam could not have passed. Contour and ruled surface maps of antenna temperature have been produced by a computer, and the results plotted on an optical display device.

A small degree of linear polarization $(1.9 \pm 0.2 \%$, at $71 \pm 5^{\circ}$ ) has been measured near the geometric center of Cassiopeia A. The distribution of antenna temperature shows a bright spot on the northwest side, a result already reported by Zisk (Zisk, S. H., Science 153, 1107, 1966).

Contours of the randomly polarized component of the radiation received from Taurus A are roughly elliptical ; the ellipse (assuming Gaussian source and antenna beam shapes) has a minor axis of $2.3 \mathrm{~min}$ of arc, and major axis $3.7 \mathrm{~min}$ of arc oriented at position angle $135^{\circ}$. Contours of the linearly polarized component arc also roughly elliptical with major axis oriented at position angle $57^{\circ}$, but the centroid is displaced $0.9 \mathrm{~min}$ of arc away to the southeast in the direction of position angle $142^{\circ}$. The polarized source is apparently not resolved along its minor axis, and is $2.1 \mathrm{~min}$ of arc in extent along its major axis (assuming Gaussian source and beam models). The linear polarization at the maximum is $11.0 \%$, at position angle $159^{\circ}$. This position angle persists over most of the polarized component.

The contours of the radio source Cygnus A are also elongated, approximately in position angle $110^{\circ}$. Perpendicular to this direction, the source is much smaller than the antenna beam. Along the major axis of the source, the position angle of the linear polarization varies from $39 \pm 5^{\circ}$ on the eastern side $\left(8.2 \pm 0.7 \%\right.$ polarization) to $147 \pm 4^{\circ}$ on the western side $(9.8 \pm 0.7 \%$ polarization). These data are not adjusted for Faraday rotation over the receiver bandwidth. The results are consistent with the model of two point sources (Ryle, M., Elsmore, B., and Neville, Nature 205, 1259, 1965), separated by $1.80 \mathrm{~min}$ of arc, and polarized in different directions.

This work is supported by the National Aeronautics and Space Adminstration Grant NsG-419. Lincoln Laboratory is operated by the Massachusetts Institute of Technology with support from the U. S. Air Force.

Time Variations in the Radio Polarization of the Quasi-Stellar Source $3 \mathrm{C} 273$ at $8000 \mathrm{Mc} / \mathrm{sec}$. H. D. Aller and F. T. Haddock, Radio Astronomy Observatory, University of Michigan.-Measurements of the polarization of 3C273, taken from July 1965 through October 1966, combined with a measurement taken in 1963 (Haddock and Hobbs 1964) with the University of Michigan $85-\mathrm{ft}$ radio telescope, show that the polarized radiation of this source varies significantly in periods of less than one year. During this time the plane of polarization 